\title{
Accelerometers: An Underutilized Resource in Sports Monitoring
}

\author{
Jonathon Neville $^{\# 1}$, Andrew Wixted ${ }^{\# 2}$, David Rowlands ${ }^{\# 3}$, Daniel James ${ }^{\#, * 4}$

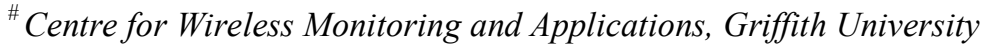 \\ Nathan, Queensland, Australia. \\ jonathon.nevilledgriffithuni.edu.au \\ ${ }^{2}$ a.wixtedegriffith.edu.au \\ ${ }^{3}$ d.rowlandsegriffith.edu. au \\ *Centre of Excellence for Applied Sports Science Research, Queensland Academy of Sport, Nathan, \\ Queensland, Australia \\ ${ }^{4}$ d.jamesegriffith.edu.au
}

\begin{abstract}
Play based sports monitoring techniques provide coaches and players with the tools to better measure the effects of training or live performance. This paper explores the advantages of using accelerometers units, in an effort to better analyse over ground running in professional athletes. A large portion of studies in player monitoring in the Australian Football League (AFL) utilize GPS to obtain time and distance measurements.

Previous studies have shown that the speed and energy expenditure of an athlete while running on a treadmill can be obtained through the use of body mounted accelerometers. This research extends these concepts by applying them to overground running data from professional players.

A study was conducted using professional AFL players and GPS and accelerometer sensors. The data obtained was filtered and the stride frequency was obtained for stepwise bands of constant running speed between $4 \mathrm{~km} / \mathrm{h}$ and $24 \mathrm{~km} / \mathrm{h}$. Stride frequency was compared to speed obtained from the GPS. A linear relationship between stride frequency and speed was identified and agreed with the literature. This indicates that a player's speed can be approximately derived from their stride frequency, and thus, more efficient and cost effective accelerometer can be used in lieu of GPS units.
\end{abstract}

\section{INTRODUCTION}

Accurate activity monitoring techniques provide coaches and players with the tools to better measure the effects of training sessions in the lead up to competition. Player workload management plays an important role in all professional sporting teams. Activity monitoring gives coaches the ability to monitor the physical demand on athletes by identifying the different activities of a training session or competition and the intensity of these activities. Activity monitoring also plays an important role in injury prevention and rehabilitation by providing information on players' current performance levels.

A large portion of studies in player monitoring in the Australian Football League (AFL) focus on obtaining the time spent in different speed levels as well as the total distance travelled [1-3]. The Global Positioning System (GPS) longitudinal and latitudinal positioning of players is rarely used as GPS tracking is used more for player speed than position. In a single game it is common for players to run between 12 and $20 \mathrm{~km}$ and loose over $3 \mathrm{~kg}$ in body mass [4]. A loss of mass of over $2 \%$ can lead to dehydration and other health concerns. It is therefore important for coaches and players to understand the workload and stress during training and game sessions.

In general GPS analysis tools are poorly used and understood. Accelerometers on the other hand are a new technology emerging in sports and provide an easy to use alternative.

This paper explores the advantages of using accelerometer based technology as an alternative to GPS units, to approximate the speed of a player running at constant speed. Current analysis techniques used to monitor speed and energy usage of professional sports men and women require the use of computationally heavy and power hungry GPS based devices. The main drain on power for a GPS based device is associated with the continuous receiving of data. Therefore, by lowering the refresh rate on most GPS devices power can be saved [5]. This, however, comes at a cost to accuracy, particularly when dealing with situations where motions are fast paced and changes in speed and direction are common. As sacrificing accuracy is not always a desired option, a solution to this problem can be through the application of accelerometers.

Research conducted by Herren, Sparti, Aminian and Schutz [6] demonstrates accelerometers can be used to obtain a participants speed. Previous studies by Wixted [7,8] have shown that the speed and energy expenditure of an athlete while running on a treadmill can be obtained through the use of body mounted accelerometers. This case study extends upon the concepts developed by the previously mentioned authors by applying them to constant speed overground running data from a professional player during training and competitive games. 


\section{Methodology}

The aim of this research is to investigate the relationship between the stride frequency of professional athletes and their GPS measured speed in relation to over ground running. This study also provides a method to extract player stride frequency from accelerometer data. The study was conducted using data gathered from an elite, first grade, Australian Football League (AFL) player from the 2009 AFL season. It includes data collected from a mix of game and training sessions (18 game sessions and 42 training sessions).

Data was collected using a GPSport's Wi SPI (1 Hz) GPS tracking unit. This unit includes a GPS sensor which logged coordinates at $1 \mathrm{~Hz}$ coupled with three axes of acceleration, which measured up to $+/-8 \mathrm{~g}$, and logged data at $100 \mathrm{~Hz}$. The GPSport's Wi SPI unit weighed approximately $100 \mathrm{~g}$ and continuously recorded data for over four hours. The data was stored locally on the device and downloaded to a computer after each training or game session through the use of a USB cable and docking station.

The tracking units were placed in a specially designed vest that when worn by the player, positioned the tracking unit around the middle to upper thoracic vertebrae. Figure 1 shows the three axis of the accelerometer with respect to the player. The three axes of accelerometer align with the player's body in such a way that acceleration in the $\mathrm{x}$ channel correspond to movement in the mediolateral axis, accelerations in the y channel correspond to movement in the superior-inferior axis and accelerations in the $\mathrm{z}$ channel correspond to movement in the anteroposterior axis.

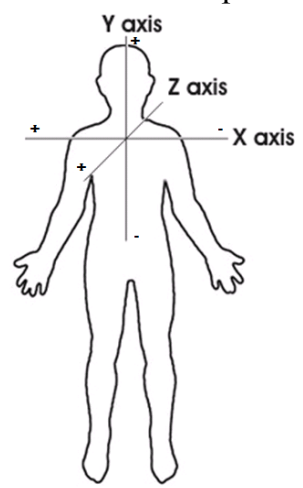

Fig.1 A visual representation of the location and orientation of the GPS, accelerometer tracking unit. (The outline depicted in the figure is facing forward - out of the page).

The vests and tracking units were worn by all players for all training sessions and most game sessions and do not impede the player's movements.

All research was conducted in accordance with the National Statement on Ethical Conduct in Human Research. Griffith University Human Research Ethics Committee Reference Number ENG/04/10/HREC.

A

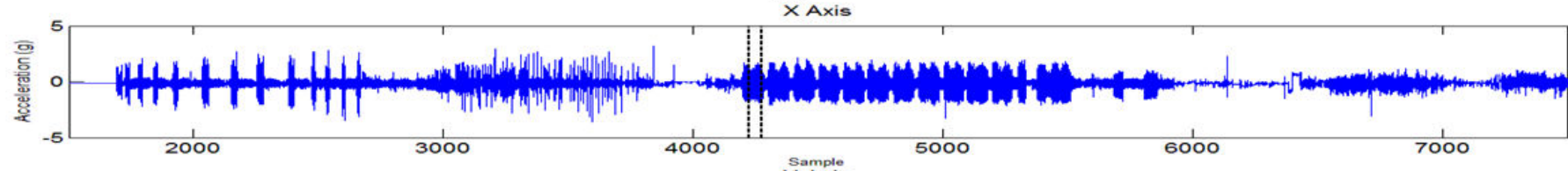

Y Axis
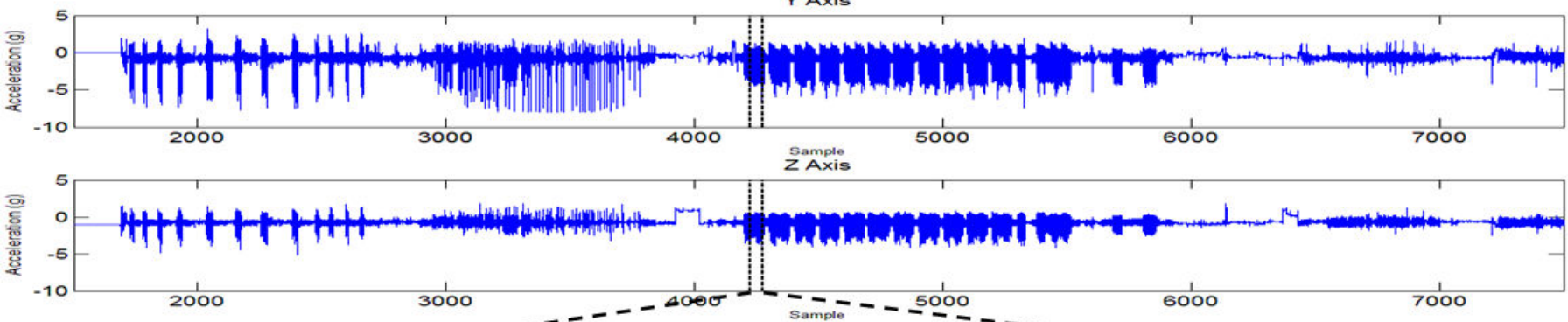

$\mathrm{B}$

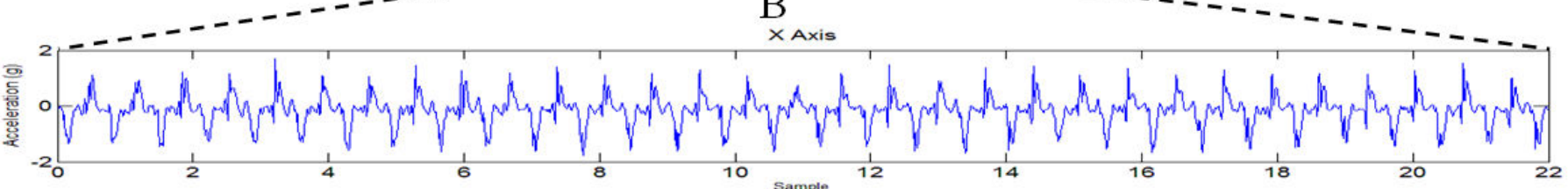

Y Axis

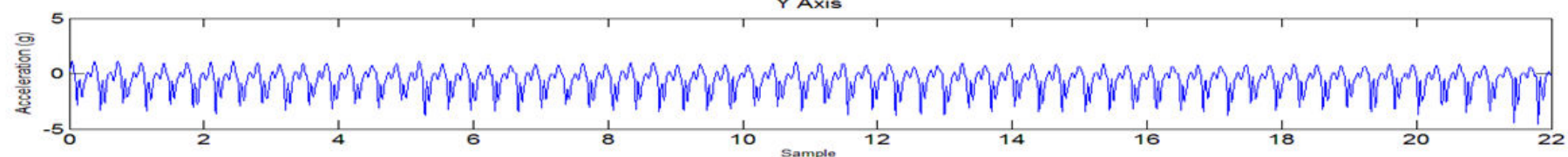

Samplo

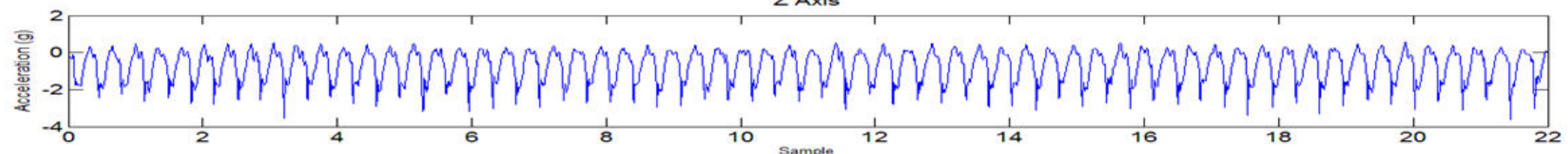

Fig2. A graphical representation of (A) the $\mathrm{x}, \mathrm{y}$ and $\mathrm{z}$ acceleration channels of a full training session of player data, and (B) the $\mathrm{x}, \mathrm{y}$ and $\mathrm{z}$ acceleration channels for section of constant speed data. 
To allow reliable determination of the stride frequency, several post processing techniques were used. Figure 3 provides a graphical view of the post processing conducted on the training and game accelerometer data in order to extract stride frequency.

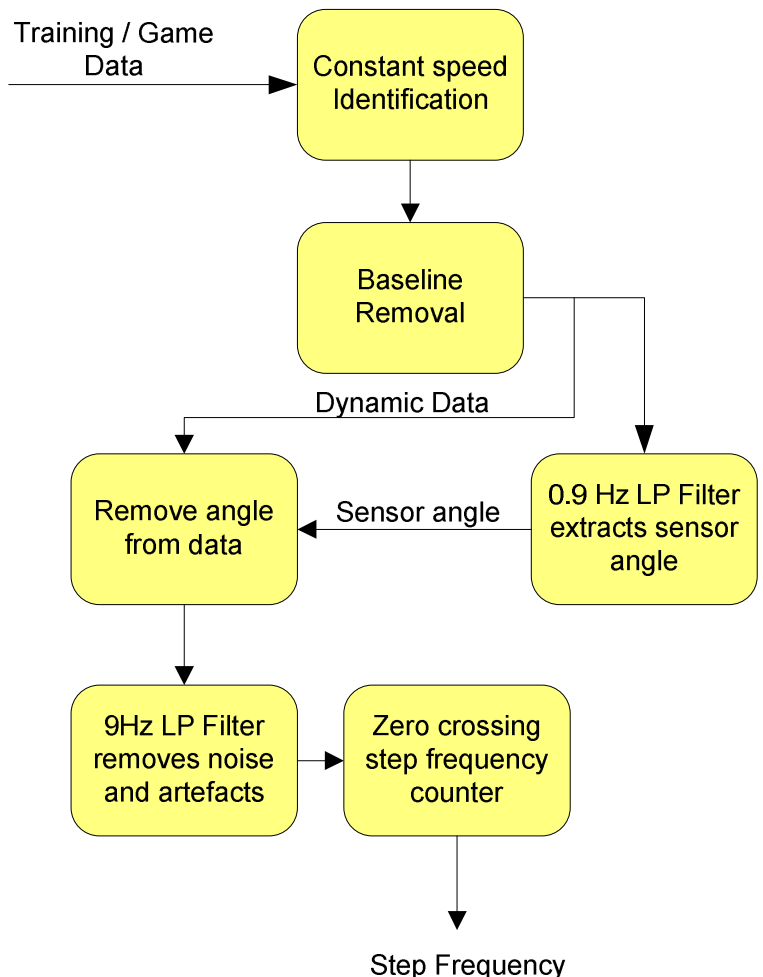

Fig.3 Post processing methodology for extracting stride frequency from accelerometer data.

The accelerometer and GPS data from each of the training sessions and official game matches was imported into MATLAB. Each individual session was stored in separate arrays consisting of accelerometer and GPS data. A program was written to sweep through each array and identify any segments of data in which the GPS calculated speed remained approximately constant $( \pm 5 \%)$ for a minimum of 15 seconds. Thus, the GPS data was used as a filter to identify intervals of constant speed. An example of the accelerometer data for a training session and an identified section of constant speed is shown in figure 2.

Figure 2 (A) is an example of data collected from a full training or game session (inactivity in the earlier samples has been removed for clarity). It can be seen in each axis, multiple events occur with different intensities of acceleration. Figure 2 (B) is an example of a single event in which the player maintains a constant speed. In this figure the player's impacts with the ground can be identified in the sharp negative acceleration spikes in the $\mathrm{y}$ axis. In the $\mathrm{z}$ axis the forward surges as a result from pushing of the ground can be seen and therefore occur at the same frequency as the ground impacts in the $\mathrm{y}$ axis. The $\mathrm{x}$ axis represents the rotation of the upper body and therefore alternates with each forward leg. In other words a positive peak in the $\mathrm{x}$ axis represents the player's right leg forward, and a negative peak in the $\mathrm{x}$ axis corresponds to the left leg forward.

The accelerometer data for each extracted constant speed section was filtered with a low pass filter of $0.9 \mathrm{~Hz}$ since 0.9 $\mathrm{Hz}$ is significantly slower than the minimum stride frequency. Therefore the resulting signal for each axis of the data represented the orientation of the sensor. The orientation signal was then subtracted from the original signal to remove any effects due to the player's angle.

Another low pass filter $(9 \mathrm{~Hz})$ was applied to the signal to remove any sharp movements or artefacts. As the maximum stride frequency for running is around $4-5 \mathrm{~Hz}$ a low pass filter of $9 \mathrm{~Hz}$ will not remove the underlying shape caused by the player's impacts with the ground. Figure 4 (A) shows the resulting data after the previously mentioned filtering and orientation processes have been performed.
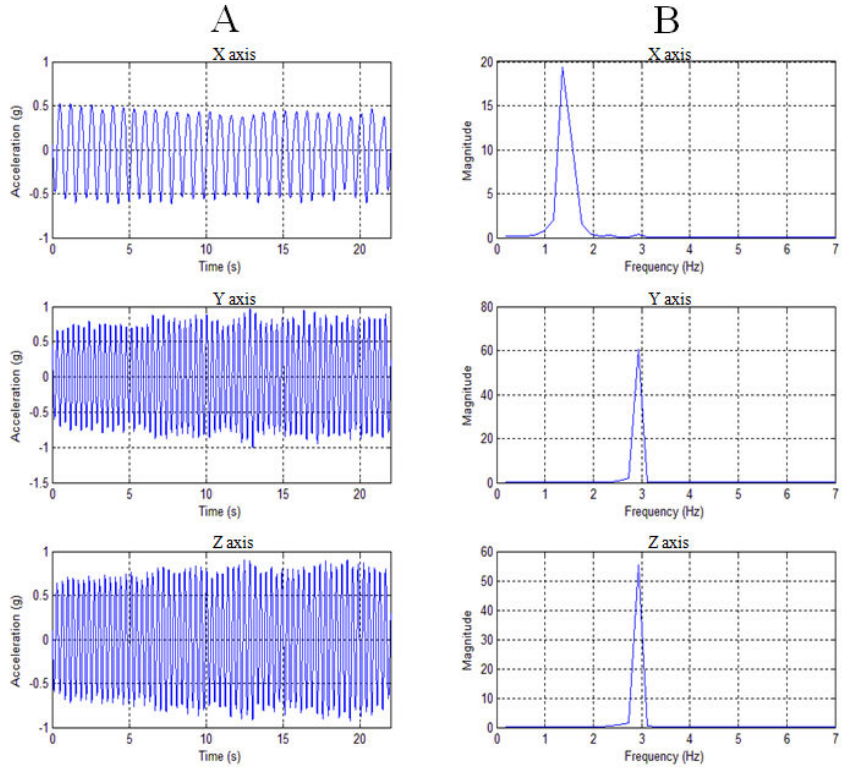

Fig.4 (A) Time domain representation of the constant speed running data after $9 \mathrm{~Hz}$ LP filter. (B) FFT of the signal in (A) provides a quick view of stride frequency.

Figure 4 (A) shows the sinusoidal waveforms that are the result of the previously mentioned filtering process. Figure 4 (B) is a Fast Fourier Transform of (A) providing a quick estimate of the stride frequency. The stride frequency used in the results was calculated using a zero crossing method. A MATLAB program was written to count the number of impacts with the ground by watching the zero crossings of the filtered signal in the $y$ axis. The number of strides in the filtered signal was then divided by the time between the first and last stride.

\section{RESULTS}

The results of the study were focused on identifying the relationship between stride frequency, measured through the use of body mounted accelerometers and the GPS calculated speed for over ground running. 


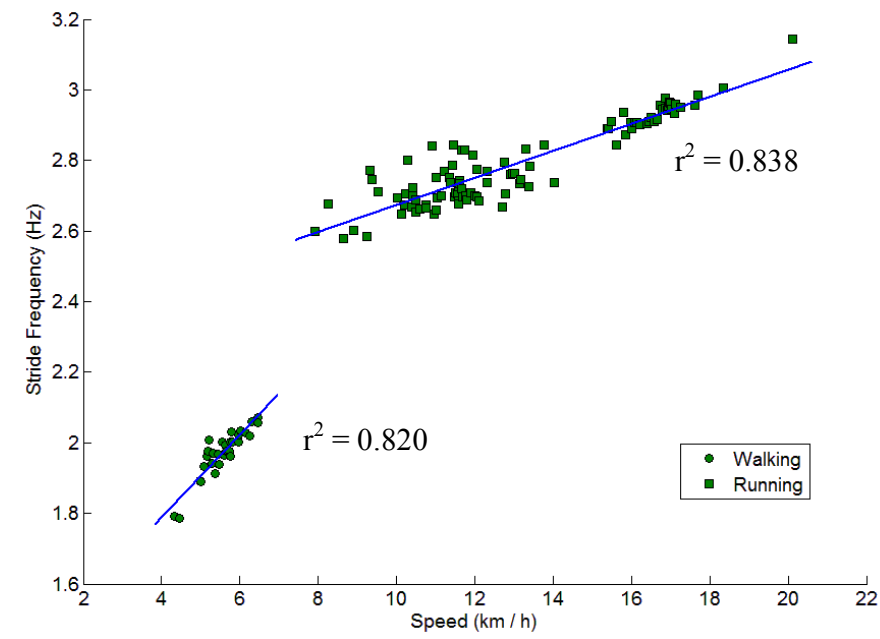

Fig.5 Graph of GPS calculated speed (x axis) and accelerometer derived stride frequency (y axis) resulting in two linear correlations. (A) Linear relationship for walking $\mathrm{r}^{2}=0.820$, (B) linear relationship for running $\mathrm{r}^{2}=$ 0.838 .

Figure 5 shows a plot of the stride frequency vs GPS speed of each of the constant speed sections $(n=131)$ ranging from $4 \mathrm{~km} / \mathrm{h}$ to $21 \mathrm{~km} / \mathrm{h}$. It included data from the player while both running and walking. The results revealed a large gap in the GPS velocity (approximately $6.5-8 \mathrm{~km} / \mathrm{h}$ ) and stride frequency (approximately 2-2.6Hz). This gap corresponds to the difference between a players comfortable walking and running speeds. Figure 5 shows two separate clusters of data; the first data subset (A) contains constant speeds in which the player is walking (approximately $4.3 \mathrm{~km} / \mathrm{h}$ to $6.5 \mathrm{~km} / \mathrm{h}$ ). A linear regression calculation was performed on the walking data and was found to support a linear relationship $\left(r^{2}=0.820\right)$. The second data subset (B) contains constant speeds in which the player is running (approximately $7.9 \mathrm{~km} / \mathrm{h}$ to $20.1 \mathrm{~km} / \mathrm{h}$ ). Again a linear regression calculation was performed and was found to support a linear relationship $\left(\mathrm{r}^{2}=0.838\right)$. It is worth noting that the results follow those of Wixted's [7] study on treadmill running.

It was observed that in the running data, larger variations in stride frequency occur at lower speeds. It is theorised that the variation in lower speed running data was due to the different activities of the player during both game and training sessions. Different activities can include ball handling drills, foot work drills and ball contention drills.

\section{CONCLUSION}

This paper considers the problems associated with accurate GPS based activity monitoring techniques. It has been identified in the literature that accelerometer based monitoring systems can provide accurate information on a players speed through the measurement of stride frequency.

The results from this study have provided a methodology for analyzing the stride frequency in constant speed running data and have shown that there is a linear relationship between speed and stride frequency for professional athletes with respect to over ground running. Future work will include addressing the estimated speed given stride frequency for multiple players.

\section{ACKNOWLEDGMENT}

The researchers would like to thank the Brisbane Lions Football Club for access to players and data.

\section{REFERENCES}

[1] B. Wisbey, P.G. Montgomery, D.B. Pyne, B. Rattray, "Quantifying movement demands of AFL football using GPS tracking", Journal of Science and Medicine in Sport, In Press, 2009

[2] A.J. Gray, D.G. Jenkins, "Match analysis and the physiological demands of Australian football" Sports Medicine, Vol. 40, no. 4, pp. 347-360, 2010 .

[3] L. Sánchez Medina, C. Pérez Caballero, "GPS applications in sport science", Archivos de Medicina del Deporte, Vol. 23, no.112, pp 143-152, March 2006.

[4] F. Sutherland, "Australian Rules Football: Facts Sheet", Sports Dietitians Australia, December 2008.

[5] Lau, et al, "GPS receiver having a low power standby mode" U.S. Patent 5,592,173, Jan 7, 1997.

[6] R. Herren, A. Sparti, K. Aminian, Y. Schutz, "The prediction of speed and incline in outdoor running in humans using accelerometry", Medicine and Science in Sports and Exercise, Vol. 31, Issue 7, pp. 1053-1059, 1999.

[7] A. Wixted, D. Thiel, D. James, A. Hahn, C. Gore, D. Pyne, "Measurement of Energy Expenditure in Elite Athletes Using MEMSBased Triaxial Accelerometers", Sensors Journal, IEEE, Vol. 7 no.4, pp. 481-488, 2007.

[8] A. Wixted, D. Billing and D. James, "Validation of trunk mounted inertial sensors for analysing running biomechanics under field conditions, using synchronously collected foot contact data", Sports Engineering, Vol. 12, no. 4, pp. 207-212, 2009. 